\title{
Shifting to automatic
}

\section{Leonardo Restivo ${ }^{1}$ and Paul W. Frankland ${ }^{1,2 *}$}

Program in Neurosciences and Mental Health, The Hospital for Sick Children, Toronto, ON, Canada

2 Department of Physiology and Institute of Medical Science, University of Toronto, Toronto, ON, Canada

*Correspondence: paul.frankland@sickkids.ca

\section{A commentary on}

Dynamic reorganization of striatal circuits during the acquisition and consolidation of a skill.

by Henry H. Yin, Shweta Prasad Mulcare, Monica R. F. Hilário, Emily Clouse, Terrell Holloway, Margaret I. Davis, Anita C. Hansson, David M. Lovinger and Rui M. Costa (2009). Nat. Neurosci. 12, 333-341.

How does the brain acquire and retain complex motor kills? In a well-known zen story, a centipede was asked how he could coordinate all of his numerous feet without stumbling. The centipede said that he had never given it a thought. From that time on, the centipede became unable to move. This story nicely illustrates the idea that many motor skills that we commonly use throughout our life are mostly automatic and not accessible to conscious recall. The benefit of this shift to automation (or habit) is that it frees us from continuously allocating attentional resources to motor sequences, making multi-tasking a possibility. However, habit formation represents only the last stage of the complex process of motor skill learning: the acquisition of motor skills is characterized by an initial phase of rapid improvement of the performance which is then followed by a later phase during which memory becomes more automatic as performance reaches an asymptotic level (Shiffrin and Schneider, 1977).

Learning new motor sequences likely depends on a wide network of brain areas, necessarily involving the interaction between sensory and motor systems (see Pennartz et al., 2009 for recent progress). The fine tuning of new motor sequences is achieved through effortful evaluation of sensory information about the outcome of the movements, and with sufficient repetition only sensory feedback is needed to link movement sequences. This two-stage process of motor skill acquisition suggests that the brain networks underlying the expression of motor performance gradually reorganize over time. Indeed, recent evidence indicates that two distinct compartments of the monkey striatum contribute differentially to the early and late stages of procedural learning (Miyachi et al., 2002). The dorsomedial region of the striatum (DMS), which is primarily innervated by association cortices, seems to be preferentially recruited during initial stages of visuo-motor learning (Miyachi et al., 2002). In contrast, the dorsolateral portion (DLS), which is primarily innervated by sensorimotor areas, is more important for the gradual acquisition of automatic behavior (Miyachi et al., 1997; Tang et al., 2007).

Building on these observations, Yin et al. (2009) now identify region specific changes in striatal neural activity that map onto different phases of skill learning. Yin and colleagues implanted mice with microelectrode arrays aimed at the DMS and the DLS and trained them in the accelerating rotarod task. The rotarod task requires the acquisition of complex movements that take both time and practice to learn. As with many new skills, performance in this task is characterized by rapid initial improvement on the first day of training, with performance asymptoting after 3 days of training. The authors used this task to finely dissect early and late phases of the skill learning process.

In vivo recordings showed a functional dissociation between striatal regions: DMS neurons showed robust rate modulation during early training phase, while DLS showed increased rate modulation during the extended training period. Accordingly, DMS excitotoxic lesions impaired skill learning when performed early during training, but had no effect when performed after extended training. In contrast, DLS lesions affected both early and late phases of training suggesting that DLS and DMS activation is required to acquire the motor skill, while the DMS is disengaged as the skill becomes more automatic.

Ex vivo recordings showed that DMS and DLS medium spiny neurons exhibited training phase-related changes in glutamatergic transmission. EPSP slope measured in response to increasing afferent stimulation in the two striatal regions revealed that the average synaptic strength was higher in DMS during early training, but increased in the DLS after extended training.

Medium spiny striatal neurons can be segregated into two distinct populations on the basis of their projections: striatonigral neurons, projecting to substantia nigra, and striatopallidal neurons which send their projections to the external globus pallidus. These two populations exhibit distinct physiological properties and express different dopaminergic receptors, with striatonigral and striatopallidal neurons preferentially expressing D1 and D2 receptors, respectively. Using D2-eGFP mice, Yin and colleagues found that D2 expressing striatopallidal neurons located in DLS exhibited a significant increase in synaptic strength in comparison to D1 expressing neurons from the same region when mice underwent extended training. Accordingly, D1 blockade had no effect on performance when injected during the extended training phase, whereas D2 blockade impaired performance at both early and late training phases. Therefore, these data suggest that skill automatization likely involves an increase in synaptic activation of D2 expressing medium spiny neurons in the DLS. Yin and colleagues also noted that D2 expressing neurons have more and stronger inhibitory projections on D1 expressing neurons than the converse (Taverna et al., 2008), a difference which might provide a mechanistic basis for neural competition between striatopallidal and striatonigral circuits during skill learning and subsequent consolidation. 
The study by Yin and colleagues brings new, compelling evidence that functional reorganization of the internal circuitry of the striatum accompanies different stages of skill learning. This finding is further supported by Kimchi et al. (2009) data showing that progressive change of DLS activity is likely to occur also during learning of an instrumental task. Such dynamic reorganization of brain circuits seems to be a fundamental property of learning systems where initial information is subjected to rapid degradation and new memory traces have to be functionally integrated with previously acquired information (McClelland et al., 1995; Frankland and Bontempi, 2005). The advantage of functional circuit reorganization resides in the capability to integrate new information into several distinct neural contexts, making the new memory accessible in various situations. Moreover, as a consequence of functional embedding of new memories into distinct neuronal networks, memory traces may eventually become less prone to degradation and more durable.

What happens to the skill memory once it has been acquired? Recent evidence shows that neural rewiring rapidly occurs in the motor cortex after training mice in the accelerating rotarod (Yang et al., 2009). These data might raise the question whether motor cortex rewiring precedes, co-occurs or is a consequence of synaptic strength changes taking place in the DLS. It is tempting to speculate that skill learning involves a three stage process where initial effortful learning is accomplished by DMS due to its connections with associative prefrontal cortices. Automatization might be subsequently achieved through a strengthening of synapses onto DLS neurons projecting to basal ganglia and thalamic structures which eventually convey information to the motor cortex where the new skill memory is embedded in a more ample, cortical-based, motor repertoire.

\section{REFERENCES}

Frankland, P. W., and Bontempi B. (2005). The organization of recent and remote memories. Nat. Rev. Neurosci. 6, 119-130.

Kimchi, E. Y., Torregrossa, M. M., Taylor, J. R., and Laubach, M. (2009). Neuronal correlates of instrumental learning in the dorsal striatum. J. Neurophysiol. $102,475-489$.

McClelland, J. L., McNaughton, B. L., and O'Reilly, R. C. (1995). Why there are complementary learning systems in the hippocampus and neocortex: insights from the successes and failures of connectionist models of learning and memory. Psychol. Rev. 102, 419-457.

Miyachi, S., Hikosaka, O., and Lu, X. (2002). Differential activation of monkey striatal neurons in the early and late stages of procedural learning. Exp. Brain Res. 146, 122-126.

Miyachi, S., Hikosaka, O., Miyashita, K., Karadi, Z., and Rand, M. K. (1997). Differential roles of monkey striatum in learning of sequential hand movement. Exp. Brain Res. 115, 1-5.

Pennartz, C. M., Berke, J. D., Graybiel, A. M., Ito, R., Lansink, C. S., van der Meer, M., Redish, A. D., Smith, K. S., and Voorn, P. (2009). Corticostriatal interactions during learning, memory processing, and decision making. J. Neurosci. 29, 12831-12838.

Shiffrin, R. M., and Schneider, W. (1977). Controlled and automatic human information processing. II Perceptual learning, automatic attending, and a general theory. Psychol. Rev. 84, 127-190.

Tang, C., Pawlak, A. P., Prokopenko, V., and West, M. O. (2007). Changes in activity of the striatum during formation of a motor habit. Eur. J. Neurosci. 25, $1212-1227$.

Taverna, S., Ilijic, E., and Surmeier, D. J. (2008). Recurrent collateral connections of striatal medium spiny neurons are disrupted in models of Parkinson's disease. J. Neurosci. 28, 5504-5512.

Yang, G., Pan, F., and Gan, W. B. (2009). Stably maintained dendritic spines are associated with lifelong memories. Nature 462, 920-924.

Yin, H. H., Mulcare, S. P., Hilário, M. R., Clouse, E., Holloway, T., Davis, M. I., Hansson, A. C., Lovinger, D. M., and Costa, R. M.(2009). Dynamic reorganization of striatal circuits during the acquisition and consolidation of a skill. Nat Neurosci. 12, 333-341.

Received: 20 December 2009; published online: 05 February 2010

Citation: Restivo L and Frankland PW (2010) Shifting to automatic. Front. Integr. Neurosci. 4:1. doi: 10.3389/neuro.07.001.2010

Copyright ( $(2010$ Restivo and Frankland. This is an open-access article subject to an exclusive license agreement between the authors and the Frontiers Research Foundation, which permits unrestricted use, distribution, and reproduction in any medium, provided the original authors and source are credited. 